\title{
ASSUMERSI LA RESPONSABILITÀ DELLE PROPRIE AZIONI: PERCHÉ Ė GIUNTO IL MOMENTO DI PENSARE ALLA STEWARDSHIP
}

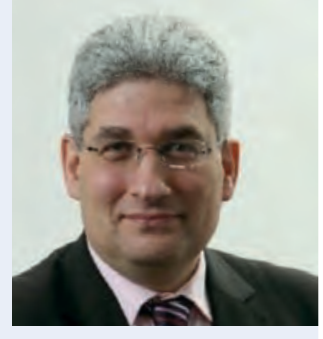

Hanno importanza le nostre azioni? Possiamo, o possono le nostre azioni fare la differenza oltre la nostra sfera di influenza? Dovremmo quindi considerare l'impatto globale delle nostre intenzioni e che senso assume ciò nell'area del project management? Questo articolo mira a incoraggiare di assumere un atteggiamento più responsabile e attento.

(traduzione e adattamento a cura di Federico Minelle)

\section{Introduzione}

In effetti siamo sempre molto presi dai nostri interessi, preferenze, priorità, problemi ecc. per darci il tempo di osservare le più ampie implicazioni e gli impatti oltre il nostro contesto più vicino. Sembra proprio che le nostre piccole decisioni e azioni provochino i loro effetti in un mondo più ampio rispetto al nostro immediato e ovvio interesse. E questo anche nel project management (PM).

Se si vuole citare un esempio "romantico" e apparentemente innocuo, un caso eclatante è quello dei cosiddetti "lucchetti dell'amore": sono quelli attaccati a un ponte, staccionata, cancello o monumento da coppie di innamorati per testimoniare il loro amore senza fine. Dopo aver inciso i loro nomi sul lucchetto, gli innamorati buttano via la chiave per simboleggiare il legame indissolubile affermato da questo gesto. Al di là della scelta del modo, quello che si è evidenziato che è questo "rito" diventato popolare (per esempio a Roma sul Ponte Milvio', a Parigi sul Pont des Arts) ha creato problemi alla stabilità degli stessi ponti, tanto che le autorità pubbliche sono dovute intervenire. Per impedire tali gesti, ad

1 Anche a seguito del romanzo Ho voglia di
te di Federico Moccia, pubblicato nel 2006 e poi trasformato in film nel 2007 esempio, Venezia ha fissato una multa di 3.000 euro.

Pertanto, questo mostra come un atto privato tra due persone finisca per impattarne molte altre.

\section{Ampliamento del nostro ambito di interesse}

Le persone sembrano essere concentrate sulle loro azioni, sui bisogni e le motivazioni, spesso ignorando le più ampie conseguenze non inserite nel loro immediato e diretto contesto. Questo rende possibili conseguenze che non sono colte da un controllo immediato, in quanto ricadono su un piano temporale più ampio rispetto al momento dell'azione e quindi non raccolgono attenzione e considerazione.

Forse una lezione importante è che dobbiamo diventare più attenti alle nostre azioni e al loro impatto, pur se siamo ben intenzionati. Se un banale lucchetto può moltiplicarsi e portare un ponte al collasso, al deterioramento dell'ambiente $\mathrm{O}$ alla distruzione di riconosciuti monumenti internazionali, forse è giunto il momento di considerare le implicazioni a lungo termine di iniziative più significative e pre-pianificate, come i progetti, i programmi e le azioni per il cambiamento.

Come gli individui sviluppano un'ossessione per la loro personalità, per l'immagine, l'ap- parenza e le azioni, diffuse tipicamente online, e dato che gli strumenti "social" incoraggiano il mettersi al centro delle cose, sia la poesia che i lucchetti possono favorire un riposizionamento del nostro interesse personale in un contesto più ampio, intenso e responsabile.

Infatti, se solo una persona avesse posto un lucchetto su un solo ponte, l'impatto complessivo sarebbe stato minimo. Ma, se ciascuno inizia a comportarsi allo stesso modo, l'impatto sulle risorse comuni non è più innocuo. Ecologisti, economisti e scienziati sociali citano questo effetto come la "tragedia dei beni comuni" [1], quando una risorsa condivisa viene distrutta come risultato di una azione di massa e viene sfruttata da molti individui che agiscono tutti indipendentemente, motivati dal loro interesse personale. L'effetto congiunto di così tante azioni collettive è di erodere, esaurire, rovinare e distruggere le risorse comuni. In questo contesto, è intesa come bene comune ogni risorsa condivisa e non regolamentata, andando dalla atmosfera ai luoghi di bellezza naturali, agli spazi aperti, fiumi, oceani, laghi, alberi, all'energia, petrolio, carbone, agli animali, stormi di uccelli e branchi di pesci, fino ai manufatti artificiali dell'uomo che includono strade, ponti, parchi e monumenti. 
I sistemi delle risorse comuni possono collassare a causa dell'uso eccessivo da parte della più ampia comunità, a meno che non sia fatto uno sforzo per regolare o governarne l'uso [2, 3]. Tale regolamentazione può essere effettuata da un'ampia comunità o gruppo, o emergere dalle azioni responsabili degli individui consapevoli.

«L'individualismo è tenuto caro perché genera libertà, ma il dono è condizionato: più la popolazione esagera nello sfruttare la capacità dell'ambiente, più si deve rinunciare alle libertà» [4, p. 683].

\section{Opportunità di una buona gestione (stewardship)}

Una alternativa al governo e alla regolamentazione imposti dall'alto può arrivare da una gestione informata, dove i membri locali interessati e impegnati cooperano e coordinano le loro azioni per evitare il collasso delle risorse comuni. La nozione di stewardship incorpora la responsabilità, una maggiore considerazione e una attenzione nel sostenere l'interesse comune.

L'Oxford Dictionary definisce la stewardship come «l'atto di prendersi cura di o gestire qualcosa, per esempio una proprietà, una organizzazione, il denaro o oggetti di valore». II Dizionario Merriam-Webster definisce la stewardship come "la conduzione, supervisione o gestione di qualcosa, specialmente la gestione attenta e responsabile di qualcosa affidata alla attenzione di qualcuno», dando così l'esempio specifico delle risorse naturali.

Così la stewardship sembra riferirsi al modo con cui si proteggono, utilizzano, condividono e gestiscono risorse speciali o una specifica capacità o valore. In termini teologici, secondo diverse religioni, è intesa come il credo che gli umani sono responsabili del mondo (o dell'universo) e quindi dovrebbero prendersene amorevolmente cura.

II termine diventò popolare con il libro di P. Block [5] pubblicato con successo nel 1993 e ristampato anche nel suo ventennale. Block definì la stewardship come «la scelta di presiedere in modo ordinato alla distribuzione del potere». Questo implica dare alle persone ai livelli più bassi e ai confini della organizzazione la scelta su come servire un cliente, un cittadino, una comunità, nel contempo riconoscendo che essi stanno svolgendo un servizio piuttosto che una funzione soggetta a controllo. In sintesi, stewardship è essere responsabili senza essere controllati o ossequienti alle regole. Nell'analizzare gli sviluppi nei 20 anni tra le due edizioni, il termine è stato riposizionato come «una scelta di agire come servizio sul lungo termine e a favore di quelli che hanno poco potere».

Block argomenta in modo convincente l'esigenza di abbandonare il tipico modello di governance gerarchico, con lo scopo di liberare l'iniziativa nelle organizzazioni e nei loro dipendenti, promuovendo la responsabilizzazione, il senso di appartenenza e la responsabilità. II cambiamento nell'approccio richiede un certo numero di modifiche sostanziali e di scelte intenzionali, individuate in:

- sostituire la leadership con la stewardship;

- scegliere la condivisione rispetto al "patriarcato";

- scegliere l'avventura rispetto alla sicurezza;

- scegliere il servizio rispetto all'interesse personale.

II cambiamento (anche dirompente) è particolarmente importante per consentire ai sistemi di management e di stewardship di affrontare le seguenti sfide:

- Fare di più con meno - mantenere la responsabilità sul pro- dotto/servizio al cliente, specialmente con minori risorse e maggiore domanda.

- Imparare ad adeguarsi alla clientela e al mercato - diventando il cliente più importante, è necessario abilitare il personale che interagisce direttamente con il cliente a rispondere alle sue esigenze, senza dover ricorrere al "responsabile gerarchico".

Generare passione e impegno nel personale - stimolare l'impegno diretto sviluppando il senso di appartenenza e di responsabilità, anche se la sicurezza del lavoro non è più garantita.

Quanto detto è certo applicabile anche al mondo dei progetti.

\section{Significato della stewardship sul prodotto}

Muoversi dall'interesse personale verso il servizio è un'idea rivoluzionaria che supera la separazione artificiale tra il fare e il gestire, come invece ideato e supportato dalla teoria e pratica dello scientific management [6]. La stewardship incoraggia gli individui ad operare oltre il loro interesse personale. Restituisce potere ai lavoratori che operano direttamente nei confronti del cliente, consente la creazione di organizzazioni con atteggiamento positivo basato sul loro potere di cambiare e trasformare [7].

Per di più, dà una maggiore enfasi al prodotto "esteso", ai risultati e agli effetti delle iniziative di cambiamento e sulle considerazioni di lungo termine relative all'intervento e ai miglioramenti durevoli per la comunità coinvolta.

Fino a poco fa quest'area non aveva goduto di grande considerazione nel lavoro progettuale; tuttavia l'ultima edizione del APM Body of Knowledge [8] evidenzia che molti progetti de- 
vono considerare la gestione del cambiamento e la realizzazione dei benefici, richiedendo quindi I'applicazione del concetto di "ciclo di vita esteso" [8, p. 24]. La considerazione dei benefici si estende oltre la consegna dei risultati definiti per il progetto, includendo l'adozione e lo svolgimento di attività addizionali e significativi conseguenti contatti con i soggetti del cambiamento. Tuttavia, è più cruciale il bisogno di assumersi la responsabilità su tutto il ciclo di vita del prodotto: dall'idea iniziale, attraverso il suo sviluppo, evoluzione e miglioramenti, fino alla rimozione dall'uso e il suo "smontaggio" [8, p. 26]. Mentre tali considerazioni si estendono oltre i confini tradizionali dei progetti, i problemi relativi alla gestione operativa, migliorie, smontaggio e smaltimento possono essere aiutati con l'applicazione di un atteggiamento di stewardship rispetto al più ampio contesto del progetto.

Nonostante ciò, poco finora si è scritto sulla applicazione del ciclo di vita del prodotto e sul sottostante bisogno di stewardship nel contesto dei lavori di progetto. Una nuova riflessione sull'impatto ambientale dei prodotti e dei progetti è essenziale per sviluppare una approfondita comprensione sulle prassi da adottare pragmaticamente in quest'area. Inoltre, come la stewardship sul prodotto è diventata più comune, vi è un fondamentale bisogno di accedere al suo contenuto e renderlo disponibile alla comunità coinvolta nel progetto.

H. Lewis [9] offre una importante introduzione alla stewardship di prodotto e descrive come e perché le imprese più avanzate si stanno assumendo la responsabilità per l'impatto ambientale dei loro prodotti e del relativo confezionamento (packaging).
Questa ricerca si basa sulla conoscenza e l'esperienza di operatori aziendali e di altri esperti, per fornire un approccio strutturato sulla responsabilità di prodotto dentro le organizzazioni. La stewardship viene vista come una miscela di azioni volontarie da parte delle organizzazioni, insieme a schemi di regole pubbliche che attengono agli impatti sociali e ambientali. Nel far questo, l'autrice estende la stewardship di prodotto oltre il confine degli schemi di regole e alla loro aderenza, per includere la responsabiltà etica e sociale per agire. La stewardship di prodotto è quindi debitamente definita come il «principio che ciascuno coinvolto nella produzione, distribuzione o consumo di un prodotto condivide la responsabilità per gli impatti ambientali e sociali di quel prodotto su tutto il suo ciclo di vita» [9, p. 5].

Questa definizione è importante in quanto va oltre la produzione (o la realizzazione del progetto) per includere altri attori quali i distributori, fornitori, venditori al dettaglio e proprietari del marchio. Più importante, include anche i consumatori, portando questi e gli utenti nel gruppo degli steward responsabili. I gruppi di progetto possono essere una parte inevitabile dell'impegno produttivo, ma devono anche essere consapevoli che la responsabilità si amplia all'intero ciclo di vita del prodotto progettuale. L'inserimento di distributori e consumatori potrebbe porre una ulteriore responsabilità sul processo produttivo e sul team di progetto, per considerare la conseguente interazione e il suo impatto e per progettare prodotti e/o progetti con queste considerazioni in mente. Una ulteriore implicazione è che la responsabilità sul prodotto si amplia oltre il rilascio formale e la messa in esercizio. Infatti non è limitata dalla disattivazione e smaltimento, ma dall'intera gamma delle azioni fattibili durante l'uso e l'impiego.

Un modello estremamente utile [9, pp. 10-11] è stato introdotto per considerare l'ambito della stewardship di prodotto su quattro aree di attività:

politiche che stabiliscano obiettivi e traguardi per la sostenibilità del prodotto;

processi di progettazione che considerino l'impatto di sostenibilità su tutto il ciclo di vita;

politiche e linee guida per gli approvvigionamenti;

recupero del prodotto alla fine del suo ciclo di vita, totalmente o parzialmente finanziato dal produttore.

La stewardship di prodotto può essere contrapposta alla Responsabilità Estesa del Produttore (EPR), che assegna la responsabiltà del prodotto, su tutto l'intero ciclo di vita, al produttore, specialmente in termini di riciclo e smaltimento. La EPR può quindi essere vista come una strategia per aggiungere $i$ costi ambientali relativi a tutto il ciclo di vita del prodotto nel suo prezzo di vendita [10]. Benché I'EPR e la stewardship di prodotto in qualche misura si sovrappongano, Lewis conclude che I'EPR punta agli aspetti obbligatori, mentre la stewardship di prodotto include anche gli aspetti volontaristici [9, p. 9], più vicini al modello di Blake.

Quindi mostra il beneficio ottenibile nel raggiungere un valore condiviso sia pubblico che commerciale, rispondendo efficacemente alle preoccupazioni degli stakeholder sull'ambiente. Questo genera un vantaggio competitivo. Alla fine diventa imperativo se si vuole garantire un futuro sostenibile per la nostra comunità e per le risorse che ci sono più care. 


\section{La rivalutazione della stewardship}

La stewardship di prodotto punta l'attenzione sul prodotto e sul suo impatto complessivo. II suo valore sta nel far sì che i produttori e manager ripensino e riconsiderino il più ampio impatto - $\mathrm{e}$ in qualche modo riprogettino o riposizionino adeguatamente il prodotto risultante. Altri autori [11] fanno notare che gli ingegneri e i manager devono considerare l'evoluzione dei bisogni e di come i loro prodotti saranno riciclati, di come i clienti useranno i loro prodotti e che rischi ambientali ne possano nascere. Considerare l'intero ciclo di vita del prodotto incoraggia la prospettiva di un processo che include l'intera "catena" produzione-utilizzo-smaltimento, conducendo quindi a un approccio decisionale più informato e responsabile in relazione al prodotto e al suo atteso utilizzo.

La stewardship di prodotto estende la responsabilità a ciascuno che sia coinvolto nella "catena del prodotto" attraverso il suo ciclo di vita esteso: a chiunque progetti, produca e venda o usi il prodotto è chiesto di assumersi la responsabilità di minimizzarne l'impatto su tutte le fasi del suo ciclo di vita esteso. In fondo, gestire un prodotto sul suo ciclo di vita esteso consente di considerare le implicazioni a lungo termine, ne diminuisce l'impatto ambientale e ne condivide il carico di responsabilità con tutti gli altri attori partecipanti.

II significato di stewardship è un importante incremento al tema sulla responsabilità, in particolare nei termini di impegno verso le future generazioni. Adottare questo punto di vista aiuta, facilita e incoraggia importanti sviluppi nel considerare quali siano le più ampie implicazioni di azioni che si estendono oltre l'interesse personale e gli atti egoistici compiuti dagli individui.
La stewardship ha il compito di proteggere e curare l'interesse pubblico. Ciò può essere fatto adottando l'impegno sui beni comuni e considerando il beneficio ancora più grande, che aggiunge una dimensione sociale a quella puramente ambientale. Si va oltre i divieti e i regolamenti per sviluppare un atteggiamento mentale più sistemico e ampio che consenta una maggiore padronanza e capacità decisionale da parte di tutti i partecipanti.

Sviluppare la stewardship richiede nuovi modi di costruire le organizzazioni, prendere le decisioni, definire le priorità ed effettuarne la gestione. Incoraggiando gli individui a considerare le implicazioni delle possibili azioni, complessivamente si può diventare più responsabili per le risorse condivise, dai ponti, alle bellezze naturali, all'acqua, gli animali e l'ambiente. Può anche creare le condizioni per una crescita nelle responsabilità e nelle decisioni di carattere sociale che si rendono necessarie per sostenere una visione di più ampio respiro. La chiave per la prosperità delle comunità future può ben essere nel sostenere le conoscenze di stewardship e le annesse capacità in tutti gli interessati, non appena si comincia a collaborare per creare e agevolare un miglior futuro comune.

\section{Nota}

Articolo tradotto e sintetizzato da "Taking responsibility for our actions: The return of stewardship" di Darren Dalcher, pubblicato nel PM World Journal, Vol. VIII, Issue VII, August 2019 - www.pmworldjournal.net - a seguito della autorizzazione dell'autore e dell'editore, ed appositamente integrato dall'autore stesso per celebrare il $10^{\circ}$ anniversario della Rivista "il Project Manager"

\section{Bibliografia}

[1] Hardin G., The tragedy of the commons - Science, 162(3859), 12431248, 1968

[2] Ostrom, E., Governing the commons: The evolution of institutions for col- lective action - Cambridge University Press, 1990

[3] Ostrom E.E., Dietz T.E., Dolšak N.E., Stern P.C., Stonich S.E., Weber E.U., The drama of the commons - Washington DC: National Academy Press, 2002

[4] Hardin G., Extensions of "The tragedy of the commons" - Science, 280(5364), 682-683, 1998

[5] Block P., Stewardship: Choosing service over self-interest - San Francisco: Berrett-Koehler Publishers, 1993/2013

[6] Dalcher D., What has Taylor ever done for us? - PM World Journal, 6(4), April, 2017

[7] Dalcher D., Beyond authority: Power to the people - PM World Journal, 8(4), May, 2019

[8] Murray-Webster R., Dalcher D., (eds.) APM Body of Knowledge, Seventh edition - Princes Risborough, Buckinghamshire: Association for Project Management (2019) https://www. apm.org.uk/body-of-knowledge/, ultimo accesso sett. 2019

[9] Lewis H., Product Stewardship in Action: The Business Case for Life-cycle Thinking - Greenleaf Publishing/ Routledge, 2017

[10] OECD Extended producer responsibility: A guidance manual for governments - Organisation for Economic Co-operation and Development, Paris: OECD Publishing, 2001

[11] Rosselot K., Allen, D.T., Life cycle concepts, Product stewardship and Green Engineering - In Allen D.T., Shonnard D.R., (eds.) Green Engineering: Environmentally Conscious Design of Chemical Processes - Upper saddle River, NJ: Prentice Hall, 2001

\section{Darren Dalcher}

Fondatore e direttore del National Center for Project Management, professore in Strategic Project Management presso la Lancaster University Management School del Regno Unito (UK), è l'editore di due serie di libri di project management pubblicate da Routledge e Capo redattore del "Wiley's Journal of Software: Evolution and Process", che riflette il suo interesse verso i collegamenti tra la tecnologia e la sua corretta gestione.

$\mathrm{Ha}$ accumulato la più grande collezione al mondo di storie di insuccessi, che usa come base per perfezionare la nozione di successo sostenibile. II suo scopo è quello di attualizzare e rinnovare la gestione e la pratica della leadership attraverso la rottura dei modelli, la condivisione di idee transdisciplinari e la co-creazione di nuove avanguardie. La sua ricerca si focalizza sul ripensamento del successo di progetto, sulla ridefinizione della nozione di agilità e sullo sviluppo di una prospettiva a più lungo termine di utilizzo, beneficio e valore di opere, sistemi e progetti.

Interessi chiave del Prof. Dalcher sono: il miglioramento della pratica del progetto, l'eccellenza del progetto, le iniziative strategiche, il change management, l'ingegneria dei sistemi, i processi decisionali, la narrazione del successo e del fallimento dei progetti, il miglioramento e lo sviluppo continuo, il futuro dell'informazione, i metodi agili e l'evoluzione dei cicli di vita.

E presente in numerosi comitati di ricerca di alto livello, tra cui l'Academic Member Advisory Group di PMI e I'APM Research Advisory Group. È consulente accademico e redattore della recente settima edizione del APM Body of Knowledge. E-mail: d.dalcher@lancaster.ac.uk 